\title{
Molecular Imaging: Methods and Protocols
}

Shah K, ed.

New York, NY: Humana Press, 250 pages, $\$ 119$

Molecular imaging techniques have advanced and become invaluable tools for molecular biology research and, to a more modest extent, clinical medicine. Molecular imaging is not limited to specific imaging methods but includes optical, scintigraphic, MRI, and MR spectroscopic systems. All these techniques use tagged probes with high affinity for molecules of interest, binding-activatable probes, and genetically engineered stable reporters for optimized target visualization. Therefore, the list of investigated biologic processes is long and continues to expand.

This handbook describes a rich variety of practical procedures and methods for diverging imaging technologies. Thirty-eight expert researchers explore the latest advances in molecular imaging, describing a rich variety of practical procedures and methods for diverging imaging methods. This book is organized into 3 sections, and the first section, dealing with imaging biochemical pathways and molecular events, has 5 chapters about bioluminescence resonance energy transfer imaging, luciferase protein complementation assays, hybrid Raman-fluorescence microscopy, labeling of mesenchymal stem cells, and quantification of micro-RNA abundance. Section II, on imaging in preclinical settings, has 7 chapters discussing the imaging fate of stem cells, MRI of brain inflammation, optical characterization of arterial apoptosis, intravital fluorescence microscopic molecular imaging of atherosclerosis, MRI of transplanted stem cells, imaging of epidermal growth factor receptor variants in glioblastomas, and fluorescence-based molecular imaging. Section III, handling clinical imaging, has 4 chapters dealing with PET of angiogenesis using integrin, amyloid imaging, myocardial molecular imaging, and dual-radionuclide brain SPECT for parkinsonian diagnosis. Composed in the highly successful "Methods in Molecular Biology" series format, each chapter contains a brief introduction, step-by-step methods, a list of necessary materials, and tips on troubleshooting and avoiding known pitfalls. Images are clear and informative.

This sufficiently detailed handbook is essential for students, research fellows, and established practitioners from different fields to become familiar with molecular imaging and incorporate it into their work. I highly recommend the book to trainees and practitioners in nuclear medicine and radiology with research interest in molecular imaging.

\author{
E. Edmund Kim \\ M.D. Anderson Cancer Center \\ 1515 Holcombe Blvd., Unit 1264 \\ Houston, TX 77030 \\ E-mail:ekim@mdanderson.org
}

COPYRIGHT (C) 2012 by the Society of Nuclear Medicine, Inc.

Published online Nov. 2, 2011.

DOI: 10.2967/jnumed.111.096008 